\title{
Neoadjuvant S-1 Therapy for Resectable Pancreatic Adenocarcinoma: Findings from Phase II of the HOPS-R01 Trial
}

\author{
Toru Nakamura ( $\nabla$ torunakamura@med.hokudai.ac.jp ) \\ Hokkaido University \\ Tsuyoshi Hayashi \\ Teine Keijinkai Hospital
}

Yasutoshi Kimura

Sapporo Medical University

Hiroshi Kawakami

Hokkaido University

Kuniyuki Takahashi

Teine Keijinkai Hospital

Hirotoshi Ishiwatari

Sapporo Medical University

\section{Takuma Goto}

Asahikawa Medical University

\section{Masayo Motoya}

Sapporo Medical University

Keisuke Yamakita

Asahikawa Medical University

Yusuke Sakuhara

Hokkaido University

\section{Michihiro Ono}

Sapporo Medical University

\section{Eiichi Tanaka}

Hokkaido Gastroenterology Hospital

\section{Makoto Omi}

Kushiro Red Cross Hospital

\section{Katsuhiko Murakawa}

Obihiro Kosei General Hospital

\section{Tomoya lida}

Muroran City General Hospital

\section{Tamaki Sakurai}


Steel Memorial Muroran Hospital

\section{Shin Haba}

NTT East Sapporo Hospital

\section{Takehiro Abiko}

Japanese Red Cross Asahikawa Hospital

\section{Yoichi ITO}

Hokkaido University

\section{Hiroyuki Maguchi}

Teine Keijinkai Hospital

\section{Satoshi Hirano}

Hokkaido University

\section{Research Article}

Keywords: neoadjuvant therapy, resectable pancreatic cancer, phase II trial

Posted Date: February 3rd, 2022

DOI: https://doi.org/10.21203/rs.3.rs-1147465/v1

License: (c) (1) This work is licensed under a Creative Commons Attribution 4.0 International License. Read Full License 


\section{Abstract \\ Background}

Although neoadjuvant therapy ( $\mathrm{Nac}$ ) is recommended for high-risk resectable pancreatic cancer ( $\mathrm{R}$ PDAC), evidence regarding specific regimes is scarce. This report aimed to investigate the efficacy of S-1 Nac for R-PDAC.

\section{Methods}

In a multicenter phase II trial, we investigated the efficacy of Nac S-1 (an oral fluoropyrimidine agent containing tegafur, gimeracil, and oteracil potassium) in R-PDAC patients. The protocol involved two cycles of preoperative S-1 chemotherapy, followed by surgery, and four cycles of postoperative S-1 chemotherapy. Two-year progression-free survival rates were the primary endpoint. Overall survival (OS) rates and median survival time (MST) were secondary endpoints.

\section{Results}

Forty-nine patients were eligible, and thirty-one patients underwent resection following Nac, as per protocol (31/49; 63.3\%). Per-protocol analysis included data from 31 patients, yielding the 2-year PFS rate of $58.1 \%$, and $2-, 3-$, and 5 -year OS rates of $96.8 \%, 54.8 \%$, and $44.0 \%$, respectively. MST was 49.2 months. Intention-to-treat analysis involved 49 patients, yielding the 2-year PFS rate of $40.8 \%$, and the 2-, 3-, and 5year OS rates of $87.8 \%, 46.9 \%$, and $33.9 \%$, respectively. MST was 35.5 months.

\section{Conclusions}

S-1 single regimen might be an option for Nac in R-PDAC; however, the high drop-out rate (36.7\%) was a limitation of this study.

\section{Introduction}

The National Comprehensive Cancer Network (NCCN) guidelines recommend upfront surgery for patients with resectable pancreatic ductal adenocarcinoma (R-PDAC) (clinical stage I or II). Neoadjuvant therapy (Nac) is also recommended for high-risk R-PDAC cases, such as those involving high levels of tumor marker, large primary tumors, weight loss, and so on [1]. However, evidence regarding specific regimens for Nac in R-PDAC is scarce, and participation in clinical trials is encouraged. A recent meta-analysis reported that the median overall survival (OS) of R-PDAC ranged from 12 to 25.3 months in upfront surgery, while the median OS associated with Nac group ranged from 10 to 50.2 months [2]. A separate meta-analysis based on intention-to-treat analyses has shown that patients with PDAC who received Nac had better long-term survival outcomes than patients who received upfront surgery (hazard ratio 
$[\mathrm{HR}]=0.66 ; 95 \%$ confidence interval [Cl]: 0.50-0.87, $\mathrm{P}=0.003$ ) [3]. In a large-scale propensity-score matched analysis, Nac with upfront surgery was associated with improved survival (median OS: 26 months vs. 21 months, $\mathrm{HR}=0.72 ; 95 \% \mathrm{Cl}$ : 0.50-0.87, P<0.01) [4]. At present, at least eight randomized trials have investigated the use of Nac for R-PDAC. However, only two trials have reported long-term outcomes associated with preoperative treatment. Thus, there is sparse evidence with respect to the administration of Nac for R-PDAC and the optimal protocol $[5,6]$.

The theoretical benefits of neoadjuvant therapy in R-PDAC are (a) early treatment of potentially metastatic disease, (b) identification of patients diagnosed with metastatic disease during treatment who can be spared surgical procedures that are unlikely to have survival benefit, and (c) delivery of chemotherapy and radiation to the primary tumor while it is in an intact, well-vascularized condition. In cases of R-PDAC, downsizing strategies to improve R0 resectability are not as important as they are in cases of borderline resectable PDAC or locally advanced PDAC. It should be noted that early treatment of potentially metastatic disease involves the same strategy as postoperative adjuvant therapy; however, only approximately $60 \%$ of patients with PDAC receive postoperative adjuvant therapy in the real world setting due to perioperative morbidity or early disease recurrence [7-9]. As such, the main purpose of Nac in the treatment of R-PDAC is the prevention of postoperative recurrence, which is consistent with postoperative adjuvant therapy. Preoperative treatment of R-PDAC does not need to reduce tumor size, as even if the local effect is weak, R0 resection is possible in cases of stable disease. Moreover, surgery can be avoided for patients diagnosed with distant metastases during the preoperative treatment period.

Given the advantages of adjuvant therapy before surgery, S-1 (an oral fluoropyrimidine agent containing tegafur, gimeracil, and oteracil potassium) was selected for use in the present study; this regimen has been associated with relatively good outcomes as postoperative adjuvant therapy. The JASPAC01 trial has shown that the $\mathrm{S}-1$ regimen is a superior adjuvant therapy to gemcitabine (Gem) in patients with RPDAC (mortality HR=0.57, 95\% Cl: 0.44-0.72, $p<0.0001$ ) [10]. In the same trial, disease recurrence in the liver was observed in $29 \%$ of patients in the Gem group and in $19 \%$ of patients in the S-1 group $(p=0.0016)$. Based on these findings, we hypothesized that S-1 might decrease the risk of micrometastasis, such as occult liver metastasis, in cases of R-PDAC. We conducted a multicenter singlearm phase II clinical trial to investigate the efficacy of S-1 Nac in patients with R-PDAC. This study is the first trial to focus on the prevention of R-PDAC recurrence using only a single oral agent for preoperative treatment.

\section{Results}

\section{Patient characteristics}

A total of 80 patients were diagnosed with R-PDAC by central review of MDCT findings, under the informed consent. We enrolled 49 patients in this trial between January 2014 and October 2015. The CONSORT study flow summary is presented in Figure 1. Patient demographic characteristics $(n=49)$ are summarized in Table 1. 
Table 1

Baseline Demographic Characteristics of Eligible Patients $(N=49)$

\begin{tabular}{|ll|}
\hline Characteristic & N (\%) \\
\hline Sex & $21(42.9)$ \\
\hline Male & $28(57.1)$ \\
\hline Female & $71(47-83)$ \\
\hline Age, median (range), years & \\
\hline ECOG PS & $27(95.9)$ \\
\hline 0 & $2(4.1)$ \\
\hline 1 & $36(73.5)$ \\
\hline Tumor location & $13(26.5)$ \\
\hline Head & $19(38.8)$ \\
\hline Body & $30(61.2)$ \\
\hline Biliary drainage & $22(7-58)$ \\
\hline Yes & $45.3(0.7-37105.0)$ \\
\hline No & \\
\hline Tumor size, median (range), mm & \\
\hline CA19-9, median (range), U/mL & \\
\hline Abbreviations: ECOG PS, Eastern Cooperative Oncology Group performance status \\
\hline djuant therapy, adverse events, and disease response
\end{tabular}

Treatment-related AEs are shown in Table 2. Preoperative therapy was well-tolerated by hematological markers, however, S-1 AEs that directly resulted in protocol discontinuation were diarrhea, nausea and vomiting. Of 5 patients who refused to continue Nac despite the absence of severe AEs, 4 patients opted for surgical resection, and 1 patient selected no other treatment. Among 49 eligible patients, a radiological partial response was observed in $11(22.4 \%)$ patients, stable disease was observed in 33 (67.3\%) patients, progressive disease was observed in 3 patients (2 liver metastases, 1 local progression), and 2 patients underwent no evaluation. Changes from the baseline tumor size and CA19-9 levels are shown in Supplemental Figure e1. 
Table 2

Adverse Events* Related to Neoadjuvant S-1 therapy ( $N=49)$

\begin{tabular}{|llll|}
\hline Adverse event & Grade 1-2, $\mathbf{n}(\%)$ & Grade 3, $\mathbf{n}(\%)$ & Grade 4, $\mathbf{n}(\%)$ \\
\hline Anemia & $13(27)$ & $1(2)$ & $0(0)$ \\
\hline Leukopenia & $12(25)$ & $2(4)$ & $0(0)$ \\
\hline Neutropenia & $10(20)$ & $4(8)$ & $0(0)$ \\
\hline Thrombocytopenia & $1(2)$ & $1(2)$ & $0(0)$ \\
\hline Fatigue & $6(12)$ & $1(2)$ & $0(0)$ \\
\hline Anorexia & $12(25)$ & $2(4)$ & $1(2)$ \\
\hline Diarrhea & $5(10)$ & $4(8)$ & $0(0)$ \\
\hline Mucositis oral & $4(8)$ & $0(0)$ & $0(0)$ \\
\hline Nausea & $3(6)$ & $2(4)$ & $0(0)$ \\
\hline Vomiting & $2(4)$ & $2(4)$ & $0(0)$ \\
\hline Hyperpigmentation & $6(12)$ & $0(0)$ & $0(0)$ \\
\hline Rash maculopapular & $4(8)$ & $0(0)$ & $0(0)$ \\
\hline Biliary tract infection & - & $3(6)$ & $1(2)$ \\
\hline Thromboembolic event & $0(0)$ & $0(0)$ & \\
\hline *Events were graded according to Common Terminology Criteria for Adverse Events (CTCAE) version \\
4.0.
\end{tabular}

\section{Surgical and pathological findings}

After completing Nac, 33 patients proceeded to surgery (Figure 1). Concurrently, 12 of 16 patients who experienced preoperative therapy failure were converted to surgery and received a pancreatectomy. The surgical and pathological findings of 31 patients who received pancreatectomy with Nac are shown in Table 3. Two patients received DP-CAR due to suspected tumor involvement in the bifurcation of the celiac and splenic artery. Two patients had lymph node metastasis around the middle colic artery, which was diagnosed as extra regional lymph nodes (M1). 
Table 3

Surgical and Pathological Outcomes in Neoadjuvant Complete Patients $(n=31)$

\section{Surgical and Pathological Outcomes}

$\mathrm{N}(\%)$

Operative procedure

SSPPD

$19(61.3)$

PD

$1(3.2)$

PPPD

$2(6.5)$

DP

$7(22.6)$

DP-CAR

$2(6.5)$

Portal vein resection

No

$25(80.6)$

Yes

$6(19.4)$

No. of retrieved lymph nodes, median (range)

$35(7-102)$

Blood loss, median (range), $\mathrm{mL}$

$440(50-2150)$

Operative time, median (range), min

$428(177-739)$

Tumor size, median (range), $\mathrm{cm}$

$2.0(0.5-4.4)$

Lymph node metastasis

$13(41.9)$

Portal vein invasion

$5(16.1)$

Arterial invasion (celiac axis or SMA)

0

Plexus invasion

$2(6.5)$

Residual tumor (R)

R0

$29(93.5)$

R1

2 (6.5)

Pathological Stage (UICC 7th )

IA

$5(16.1)$

IB

0

IIA

$13(41.9)$

IIB

11 (35.5)

III

0

IV

$2(6.5)$ 


\section{Surgical and Pathological Outcomes}

Pathological response (Evans Classification)

$$
\text { I }
$$

Ila

$\mathrm{Ilb}$

III

IV
$N(\%)$

$12(38.7)$

$6(19.4)$

$1(3.2)$

0

Abbreviations: SSPPD, subtotal stomach-preserving pancreaticoduodenectomy; PD, pancreaticoduodenectomy; PPPD, pylorus-preserving pancreaticoduodenectomy; DP, distal pancreatectomy; DP-CAR, distal pancreatectomy with en-bloc celiac axis resection; SMA, superior mesenteric artery; UICC, Union for International Cancer Control.

The postoperative complications after pancreatectomy are shown in Table 4. Abdominal bleeding from the right gastric artery was found in a patient who received reoperation in POD3, however there were no cases of either grade IV or V complications. The surgical and pathological results of patients receiving off-protocol resection $(n=12)$ are shown in Supplemental Table e1 and Table e2. There were no cases of either grade IV or $\mathrm{V}$ complications in the off-protocol resections. 
Table 4

Postoperative Complications after Resection (Neoadjuvant Therapy Complete Patients: $\mathrm{n}=31$ )

\begin{tabular}{|c|c|c|c|c|c|c|c|}
\hline Clavien-Dindo Classification & $\begin{array}{l}\mathrm{I}, \mathrm{n} \\
(\%)\end{array}$ & $\begin{array}{l}\text { II, n } \\
(\%)\end{array}$ & $\begin{array}{l}\text { IIla, n } \\
(\%)\end{array}$ & ${ }_{(\%)}^{\text {lllb, n }}$ & $\begin{array}{l}\text { IVa, n } \\
\text { (\%) }\end{array}$ & $\begin{array}{l}\text { IVb, n } \\
(\%)\end{array}$ & $\begin{array}{l}\text { V,n } \\
(\%)\end{array}$ \\
\hline Pancreatic fistula & $\begin{array}{l}1 \\
(3.2)\end{array}$ & $0(0)$ & $3(9.7)$ & $0(0)$ & $0(0)$ & $0(0)$ & $0(0)$ \\
\hline Delayed gastric emptying & $0(0)$ & $0(0)$ & $1(3.2)$ & $0(0)$ & $0(0)$ & $0(0)$ & $0(0)$ \\
\hline Wound infection & $\begin{array}{l}1 \\
(3.2)\end{array}$ & $\begin{array}{l}3 \\
(9.7)\end{array}$ & $0(0)$ & $0(0)$ & $0(0)$ & $0(0)$ & $0(0)$ \\
\hline Intra-abdominal abscess & $0(0)$ & $\begin{array}{l}2 \\
(6.5)\end{array}$ & $2(6.5)$ & $0(0)$ & $0(0)$ & $0(0)$ & $0(0)$ \\
\hline Bile leakage & $0(0)$ & $0(0)$ & $2(6.5)$ & $0(0)$ & $0(0)$ & $0(0)$ & $0(0)$ \\
\hline Abdominal bleeding & $0(0)$ & $0(0)$ & $0(0)$ & $1(3.2)$ & $0(0)$ & $0(0)$ & $0(0)$ \\
\hline Cholangitis & $0(0)$ & $\begin{array}{l}1 \\
(3.2)\end{array}$ & $0(0)$ & $0(0)$ & $0(0)$ & $0(0)$ & $0(0)$ \\
\hline Chylous ascites & $0(0)$ & $\begin{array}{l}1 \\
(3.2)\end{array}$ & $0(0)$ & $0(0)$ & $0(0)$ & $0(0)$ & $0(0)$ \\
\hline Diarrhea & $0(0)$ & $\begin{array}{l}1 \\
(3.2)\end{array}$ & $0(0)$ & $0(0)$ & $0(0)$ & $0(0)$ & $0(0)$ \\
\hline Portal vein embolism & $0(0)$ & $\begin{array}{l}1 \\
(3.2)\end{array}$ & $0(0)$ & $0(0)$ & $0(0)$ & $0(0)$ & $0(0)$ \\
\hline Cerebral infarction & $0(0)$ & $\begin{array}{l}1 \\
(3.2)\end{array}$ & $0(0)$ & $0(0)$ & $0(0)$ & $0(0)$ & $0(0)$ \\
\hline Intestinal bleeding & $0(0)$ & $0(0)$ & $1(3.2)$ & $0(0)$ & $0(0)$ & $0(0)$ & $0(0)$ \\
\hline $\begin{array}{l}\text { Postoperative hospital stay, days } \\
\text { (range) }\end{array}$ & \multicolumn{7}{|c|}{$21(12-67)$} \\
\hline
\end{tabular}

\section{Adjuvant therapy}

Of 31 patients who completed Nac followed by R0/R1 resection, 28 (90.3\%) started S-1 adjuvant therapy and 3 patients did not ( 2 patients had poor PS and 1 patient had bone metastasis before adjuvant therapy). Twenty-two patients completed all pre- and postoperative therapies as per study protocol ( $45 \%$ of 49 eligible patients and $71 \%$ of completed Nac patients). Meanwhile, among 12 patients who discontinued Nac but received R0/R1 resection, 8 (66.7\%) patients started adjuvant therapy and 6 patients completed it. Another 4 patients refused any chemotherapy.

\section{Survival analysis}


The 2-year PFS rate was $58.1 \%$ in 31 patients who completed Nac (per-protocol) followed by R0/R1 resection, and $40.8 \%$ in the intension-to-treat (ITT) analysis that included all 49 eligible patients (Figure 2). The primary endpoint of this trial exceeded both the expected $(48.6 \%)$ and threshold $(28.9 \%)$ values in per-protocol patients; however, the outcome of the ITT analysis fell between the expected and threshold values. The 2-year PFS rate for 18 patients who did not complete Nac (off-protocol) was $11.1 \%$. As reference data, 28 patients who had been start adjuvant S-1 therapy after R0 resection are presented in Supplemental Figure e2. The 2-year RFS and 5-year OS rates were $57.1 \%$ and $49.5 \%$, respectively, and superior to those reported by the S-1 arm of JASPAC01 study ( $48.6 \%$ and $44.1 \%$ respectively).

ITT values for the 2-, 3-, and 5-year OS rates of 49 eligible patients were $71.4 \%, 46.9 \%$, and $33.9 \%$, respectively. The MST was 35.5 months. The observed 2-, 3-, and 5-year OS rates for per-protocol patients $(n=31)$ were $80.7 \%, 54.8 \%$, and $44.0 \%$, respectively, and the MST was 49.2 months. The OS rates for offprotocol patients $(n=18)$ were $55.6 \%, 38.9 \%$, and $11.1 \%$, respectively, and the MST was 27.6 months (Figure 3). OS values for patients with off-protocol resection $(n=12)$ were $66.7 \%, 50.0 \%$, and $16.7 \%$, respectively, with an MST of 34.9 months. The OS rates for patients not undergoing resection $(n=6)$ were $33.3 \%, 16.7 \%, 16.7 \%$, respectively, with an MST of 15.6 months (Supplemental Figure e3). The median duration of follow-up was 35.5 months (range: 2.8-73.1 months) for all patients in this trial.

\section{Recurrence}

First recurrence sites (includes patients with multi-site recurrences) are shown in Supplemental Table e3. Of the per-protocol (Nac complete) resection patients $(n=31), 18$ (58.1\%) patients experienced disease recurrence; this rate was lower than that of the off-protocol (Nac failure) resections (10/12; 83.3\%). The rate of distant metastasis was high among off-protocol resections (75\% vs. $48.3 \%$ ). Liver recurrence (disease progression) for per-protocol resections, off protocol resections, and non-resection was found in $25.8 \%, 33.3 \%$, and $50 \%$ of cases, respectively.

\section{Discussion}

This trial showed that the 2-year PFS rate was $58.1 \%$ among 31 patients who completed Nac before receiving R0/R1 resection (per-protocol). In the per-protocol analysis, the primary endpoint yielded values that were better than expected. However, in the ITT analysis of 49 patients, including those who failed the protocol, the 2-year PFS rate was $40.8 \%$, which was below the reference value. Trials of adjuvant therapy such as the JASPAC01 study have reported better results than those of the ITT analysis, due to the exclusion of patients with metastasis confirmed during surgery or those with poor PS after resection and at the time of adjuvant therapy initiation [3]. In fact, the present study subpopulation that met the JASPAC01 trial criteria showed relatively better survival (Supplemental Figure e2). A recent meta-analysis, including 18 studies that involved 857 patients, has reported that the MST associated with Nac for patients with R-PDAC was 18.2 months (range: 10-50.2 months) [2]. In our trial, the MST of all 49 patients in the ITT analysis was 35.5 months, suggesting that survival outcomes in the present study were better than those in the studies included in the meta-analysis. 
At present, the evidence level for Nac in R-PDAC remains low and the optimal protocol remains unknown. At least eight trials have compared the role of neoadjuvant treatment with that of upfront surgery in the outcome of R-PDAC, and their preoperative protocols vary (Supplemental Table e4). Of these, the PREOPANC trial was the first randomized phase III trial to publish findings on the use of Nac for PDAC (including both borderline resectable and resectable); a preplanned subgroup ITT analysis demonstrated superior OS for Nac patients with BR-PDAC ( $\mathrm{HR}=0.62,95 \% \mathrm{Cl}$ : 0.40-0.95, $\mathrm{p}=0.029)$, but not for Nac patients with R-PDAC (HR=0.96, 95\% Cl: 0.64-1.44, $p=0.830)$ [5]. The subgroup settings for R-PDAC in the PREOPANC trial might be under-powered for analysis (65 Nac vs. 68 upfront surgery); furthermore, the median OS was 14.6 months in the Nac group, which was a disappointing finding. Nevertheless, the Prep02/JSAP-05 trial reported in preliminary findings that a significant benefit was observed with Nac compared with upfront surgery (median OS: 36.7 vs. 26.6 months; $\mathrm{HR}=0.72 ; 95 \% \mathrm{Cl}: 0.55-0.94, \mathrm{p}=0.015$ ) [6]. At present, no other trials have delivered high-quality evidence on the impact of Nac on R-PDAC compared with upfront surgery.

The estimated MST of the ITT analysis of our Nac S-1 monotherapy was 35.5 months, while the estimated MST in the ITT analysis of the Nac Gem plus S-1 (GS) patients in the Prep-02/JSAP-05 was 36.7 months, with no difference in survival. No data were available for comparing S-1 with GS in R-PDAC survival; however, the GEST study, which was a randomized three-arm phase III study for advanced pancreatic cancer, showed non-inferiority of S-1 but did not show superiority of GS to Gem alone for OS $[11,12]$. GS treatment was associated with a better tumor shrinkage effect than either Gem alone or S1 alone, although there was no advantage in survival. The treatment efficacy in the locally advanced disease had the advantage of GS in response rate, PFS, and OS over GEM alone [13]. The aim of Nac for $\mathrm{R}-\mathrm{PDAC}$ is to prevent metastatic recurrence rather than local control, as R0 resection can be achieved even if no tumor shrinkage. In the present study, treatment failure due to local progression was found in a single case (resected after protocol failure), and the R0 resection rate among the resected cases was $93 \%$ (40/43), suggesting that preoperative adjuvant therapy with S-1 monotherapy can achieve local tumor control in R-PDAC.

In this study, Nac was well-tolerated from the viewpoint of hematological markers; however, the gastrointestinal toxicity rate was high $(4 / 16 ; 25 \%)$. S-1 is associated with a risk of gastrointestinal toxicities, which are generally higher in Caucasian than in Asian populations due to differences in pharmacokinetics and pharmacodynamics [14]. The present trial included patients with an Asian background. However, pharmacokinetic and pharmacodynamic profile examination was outside of the scope of the present study; thus, the exact reasons behind protocol failures associated with gastrointestinal toxicity remain unclear. We speculate that patients and physicians might be concerned about undergoing surgery when even minor gastrointestinal toxicities present, as pancreatic resection is a major surgery and requires a cautious approach. An advantage of S-1 therapy is that it is a single oral agent that does not require intravenous treatment or frequent outpatient visits, thereby preserving medical resources. Nevertheless, the risk of gastrointestinal symptoms is high. In addition, this treatment might not be suitable for use in non-Asian populations. 
The second leading factor for Nac failure in the present study was patient refusal to continue with treatment despite the absence of severe AEs. In fact, patients were more likely to select surgical resection than to continue with Nac. As surgical resection is the only curative treatment for R-PDAC, patients might be eager to avoid tumor progression, which would make them ineligible for surgery. In fact, patients who refused to continue Nac had a strong desire for resection; 4 of 5 patients proceeded to surgery after discontinuing Nac. Future trials should present evidence to patients considering Nac discontinuation, and patients should be informed about the importance of completing Nac in the absence of AEs rather than immediately undergoing surgery.

In conclusion, S-1 neoadjuvant therapy for R-PDAC is safe and promising. S-1 monotherapy can be used as neoadjuvant therapy for patients with R-PDAC. However, well-designed, randomized controlled trials are required to better understand the safety profile and efficacy of this approach.

\section{Methods}

\section{Trial design and treatment}

This study was a multicenter, open-label, single-arm phase II trial of Nac S-1 in patients with R-PDAC, conducted by the Hokkaido Pancreatic Cancer Study Group (HOPS) [15, 16] (HOPS-R01 trial: University Hospital Medical Information Network Clinical Trials Registry [UMIN-CTR] number UMIN000013031, the date of first registration of the trial was 31/01/2014).

The Nac protocol involved two cycles of $40 \mathrm{mg}$ of oral S-1 for a body-surface area of $<1.25 \mathrm{~m}^{2}, 50 \mathrm{mg}$ for a body-surface area of 1.25-1.5 $\mathrm{m}^{2}$, or $60 \mathrm{mg}$ for a body-surface area of $>1.5 \mathrm{~m}^{2}$, administered twice per day for 28 consecutive days, followed by a 14-day rest period (one cycle). After completing Nac, all patients underwent dynamic multidetector computed tomography (MDCT) for restaging. All patients eligible for pancreatic resection underwent surgery 2-6 weeks after completing Nac. Patients with distant metastasis or locally advanced disease were excluded from this study, with further treatment at the discretion of the attending physician. All patients with R0/R1 surgical resection received 4 cycles of adjuvant S-1 therapy, which followed the same protocol as Nac. After completing therapy, all patients were followed up once every 3 months during the first 2 years, and once every 6 months from year 3 onwards. Tumor markers and MDCT of the chest/abdomen/pelvis or gadolinium ethoxybenzyl diethylenetriamine pentaacetic acid enhanced magnetic resonance imaging (EOB-MRI) were monitored during the follow-up period, which ended 2 years after the enrollment of the last patient.

\section{Patient population}

Central review of diagnostic imaging was performed according to the definition of the NCCN guidelines 2012 (version 2) by a radiologist (YS) and verified by a surgeon (TN) and a physician (HK). Inclusion criteria: cytologically or histologically confirmed PDAC; age $\geq 20$ years; Eastern Cooperative Oncology Group Performance Status (PS) of 0 to 1 ; sufficient dietary intake; satisfactory levels of blood parameters (white blood cell count $\geq 3500 / \mathrm{mm}^{3}$ and $<12000 / \mathrm{mm}^{3}$, neutrophil count $\geq 2000 / \mathrm{mm}^{3}$, hemoglobin $\geq 9.0$ 
$\mathrm{g} / \mathrm{dL}$, platelet count $\geq 100,000 / \mathrm{mm}^{3}$, total bilirubin $\leq 2.0 \mathrm{mg} / \mathrm{dL}$ [ $\leq 3.0 \mathrm{mg} / \mathrm{dL}$ in patients with biliary drainage], aspartate transaminase and alanine aminotransferase $\leq 100 \mathrm{IU}$ [ $\leq 150 \mathrm{IU}$ in patients with biliary drainage], creatinine $\leq 1.2 \mathrm{mg} / \mathrm{dL}$, creatinine clearance estimate by Cockcroft-Gault equation $\geq 50$ $\mathrm{mL} / \mathrm{min}$ ). Exclusion criteria: history of S-1 treatment; history of PDAC treatment; another simultaneous or metachronous (within 3 years) cancer; current use of flucytosine, phenytoin or warfarin; watery diarrhea; pulmonary fibrosis or intestinal pneumonia; confirmed or suspected pregnancy in women.

Preoperative treatment-related adverse events (AEs) were assessed using the Common Terminology Criteria for Adverse Events (version 3.0). Surgical resection was performed by laparotomy and regional lymph node dissection was required. Resection of the portal vein/superior mesenteric vein was allowed. A distal pancreatectomy with en bloc celiac axis resection (DP-CAR) due to suspected tumor involvement in tumor proximity to the bifurcation of the celiac and splenic artery was allowed [17]. Surgical morbidity was evaluated based on the Clavien-Dindo classification [18]. Pancreatic fistula was evaluated according to the classification of the International Study Group of Pancreatic Surgery [19]. Pathology findings were assessed by pathologists at each participating hospital, using UICC-TNM version 7 and the Evans classification [20]. The study protocol was approved by the Institutional Review Board of each participating hospital and the study adhered to the Declaration of Helsinki (IRB No. 013-0059, Institutional Review Board of Hokkaido University Hospital, the date of first registration was 14/02/2014, the registration number was HOPS-R01-01).

\section{Endpoints and statistical analysis}

The primary endpoint was 2-year progression-free survival (PFS). Secondary endpoints included OS, resection, and response rates, measured according to RECISTV1.1, pathological outcomes, preoperative treatment-related AEs, and surgical morbidity rate. PFS was defined as the time from registration with the trial to the date of first recurrence or disease progression, either local, distant, or both, whichever occurred first. Recurrence was defined as a radiologically rather than elevation of CA19-9. OS was defined as time from registration to the date of death from any cause and censored on the date of the final confirmation of survival for surviving patients. It was estimated using the Kaplan-Meier method.

To calculate the desired sample size for the present study, the threshold and expected values of the 2-year PFS rates were set at $29 \%$ and $48 \%$, respectively. These estimates were based on the JASPAC01 study findings, where 2-year RFS was $48 \%$ in the S-1 group and $29 \%$ in the Gem group in an adjuvant setting [10]. Given this threshold (29\%) and expected 2-year PFS (48\%), the sample size was calculated as 46, based on the Southwest Oncology Group one arm binomial tool, with a significance level of 0.025 and power of $80 \%$. In anticipation of loss to follow-up, we expected to enroll 50 patients in the present study.

\section{Declarations}

\section{ACKNOWLEDGEMENTS}


We thank the doctors who participated in data collection for their collaboration during this trial. The authors have no conflict of interest to declare. This study received no specific funding.

\section{References}

1. National Comprehensive Cancer Network (2019) NCCN Clinical Practice Guidelines in Oncology Pancreatic Adenocarcinoma, version 3. Available at: https://www.nccn.org/professionals/physician_gls/pdf/pancreatic.pdf.7. Accessed July 2, 2019.

2. Versteijne E, Vogel JA, Besselink MG, et al (2018) Meta-analysis comparing upfront surgery with neoadjuvant treatment in patients with resectable or borderline resectable pancreatic cancer. $\mathrm{Br} \mathrm{J}$ Surg 105:946-958

3. Unno M, Hata T, Motoi F (2019) Long-term outcome following neoadjuvant therapy for resectable and borderline resectable pancreatic cancer compared to upfront surgery: A Meta-Analysis of comparative studies by intention-to-treat analysis. Surg Today 49:295-299

4. Mokdad AA, Minter RM, Zhu H, et al (2017) Neoadjuvant therapy followed by resection versus upfront resection for resectable pancreatic cancer: A propensity score matched analysis. J Clin Oncol 35:515-522

5. Versteijne E, Suker M, Groothuis K, et al (2020) Preoperative chemotherapy versus immediate surgery for resectable and borderline resectable pancreatic cancer: Results of the Dutch randomized phase III PREOPANC trial. J Clin Oncol 38:1763-1773

6. Unno M, Motoi F, Matsuyama Y, et al (2019) Randomized phase II/III trial of neoadjuvant chemotherapy with gemcitabine and S-1 versus upfront surgery for resectable pancreatic cancer (Prep-02/JSAP-05). J Clin Oncol 42:190-194

7. Mayo SC, Gilson MM, Herman JM, et al (2012) Management of patients with pancreatic adenocarcinoma: National trends in patient selection, operative management, and use of adjuvant therapy. J Am Coll Surg 214:33-45

8. Merkow RP, Bilimoria KY, Tomlinson JS, et al (2014) Postoperative complications reduce adjuvant chemotherapy use in resectable pancreatic cancer. Ann Surg 260:372-377

9. Tzeng CWD, Coa HST, Lee JE, et al (2014) Treatment sequencing for resectable pancreatic cancer: Influence of early metastases and surgical complications on multimodality therapy completion and survival. J Gastrointest Surg 18:16-24

10. Uesaka K, Boku N, Fukutomi A, et al (2016) Adjuvant chemotherapy of S-1 versus gemcitabine for resected pancreatic cancer: a phase 3 , open-label, randomised, non-inferiority trial (JASPAC01). Lancet 388:248-257

11. Ueno $\mathrm{H}$, loka T, Ikeda M, et al (2013) Randomized phase III study of gemcitabine plus S-1, S-1 alone, or gemcitabine alone in patients with locally advanced and metastatic pancreatic cancer in Japan and Taiwan: GEST study. J Clin Oncol 31:1640-1648 
12. Okusaka T, Miyakawa H, Fujii H, et al (2017) Updated results from GEST study: a randomized, threearm phase III study for advanced pancreatic cancer. J Cancer Res Clin Oncol 143:1053-1059

13. Yanagimoto $H$, Ishii $H$, Nakai $Y$, et al (2014) Improved survival with combined gemcitabine and S-1 for locally advanced pancreatic cancer: pooled analysis of three randomized studies. J Hepatobiliary Pancreat Sci 21:761-766

14. Chuah B, Goh BC, Lee SC, et al (2011) Comparison of the pharmacokinetics and pharmacodynamics of S-1 between Caucasian and East Asian patients. Cancer Sci 102:478-483

15. Hayashi T, Nakamura T, Kimura Y, et al (2019) Phase 2 study of neoadjuvant treatment of sequential S-1-based concurrent chemoradiation therapy followed by systemic chemotherapy with gemcitabine for borderline resectable pancreatic adenocarcinoma (HOPS-BR 01). Int J Radiat Oncol Biol Phys 105:606-617

16. Kimura Y, Nakamura T, Hayashi T, et al (2019) Clinical usefulness of conversion surgery for unresectable pancreatic cancer diagnosed on multidetector computed tomography imaging: Results from a multicenter observational cohort study by the Hokkaido Pancreatic Cancer Study Group (HOPS UR-01). Ann Gastroenterol Surg 3:523-533

17. Nakamura T, Hirano S, Noji T, et al (2016) Distal pancreatectomy with en bloc celiac axis resection (modified appleby procedure) for locally advanced pancreatic body cancer: A Single-center review of 80 consecutive patients. Ann Surg Oncol 23:969-975

18. Dindo D, Demartines N, Clavien PA (2004) Classification of surgical complications: A new proposal with evaluation in a cohort of 6336 patients and results of a survey. Ann Surg 240:205-213

19. Bassi C, Marchegiani G, Dervenis C, et al (2017) The 2016 update of the International Study Group (ISGPS) definition and grading of postoperative pancreatic fistula: 11 years after. Surgery 161:584591

20. Evans DB, Rich TA, Byrd DR, et al (1992) Preoperative chemoradiation and pancreaticoduodenectomy for adenocarcinoma of the pancreas. Arch Surg 127:1335-1339

\section{Figures}


Figure 1

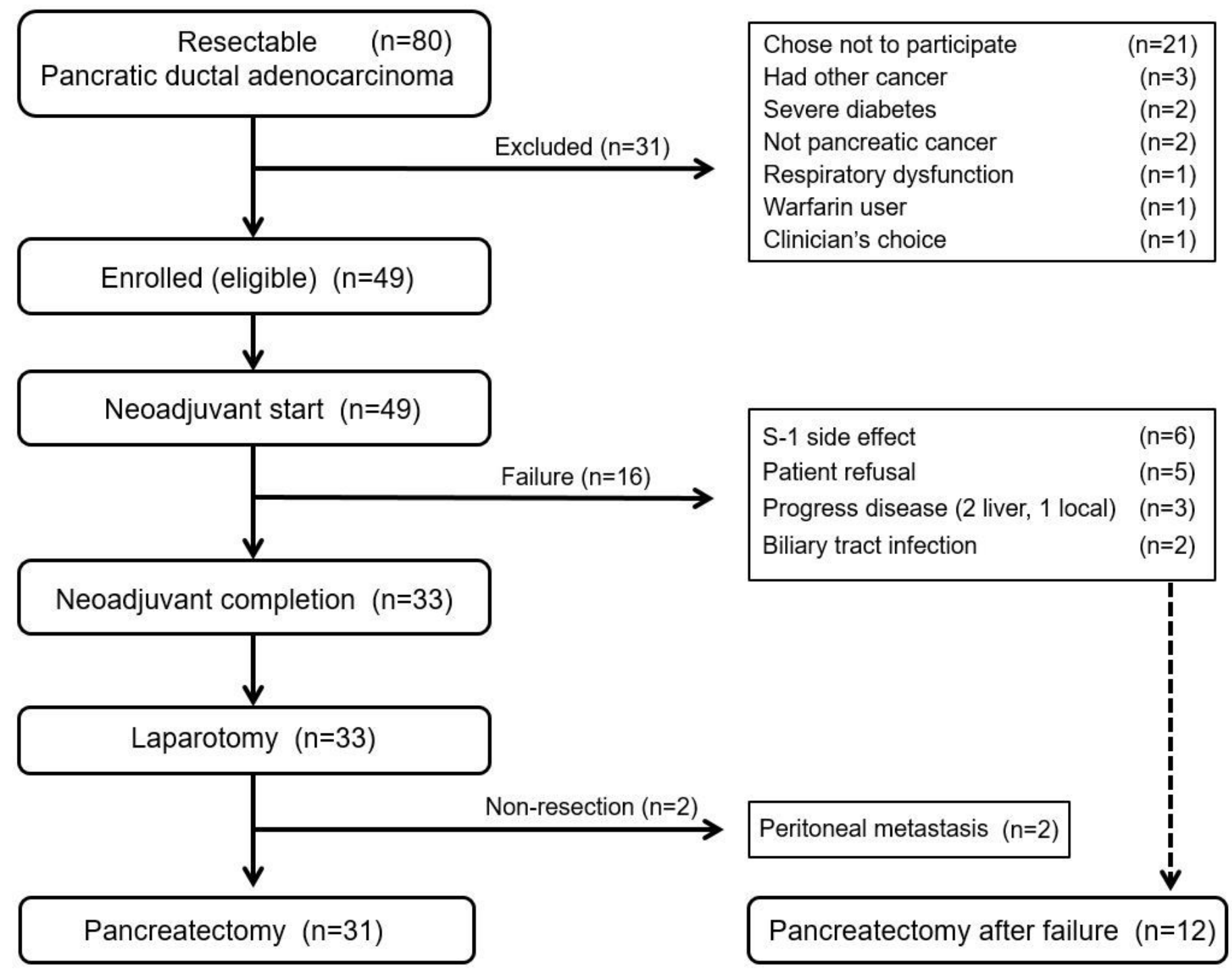

Figure 1

CONSORT diagram of the study flow.

A total of 80 patients gave informed consent for the HOPS-R01 trial between January 2014 and October 2015 and were diagnosed with R-PDAC by a central review of multidetector computed tomography.

Neoadjuvant ( $\mathrm{Nac}$ ) S-1 at $80 \mathrm{mg} / \mathrm{m}^{2}$ per day was administered for 28 consecutive days followed by a 14day rest (one cycle). The administration of S-1 was repeated every 6 weeks for two cycles. Of 49 patients who started Nac, 33 patients completed Nac and 31 patients received pancreatectomy. Of 16 Nac-failure patients, 12 patients underwent resection (pancreatectomy) 
Figure 2

(A)

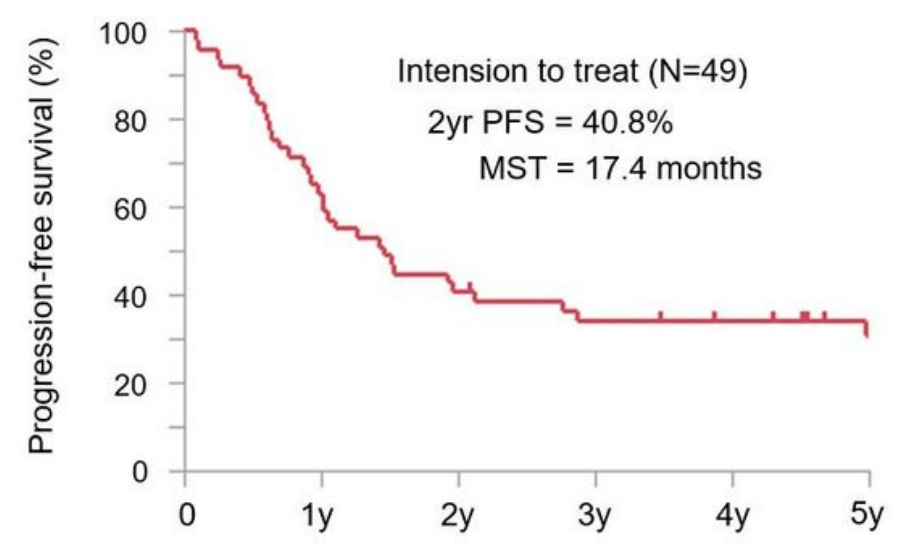

(B)

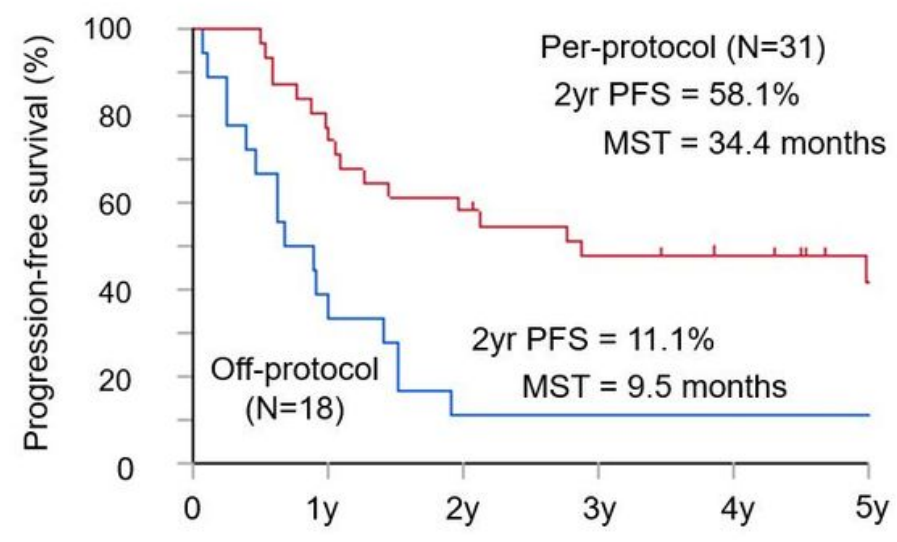

$\begin{array}{ccccccc}\text { No. at risk } & 31 & 24 & 18 & 14 & 12 & 7 \\ \% & 100 & 77.4 & 58.1 & 47.8 & 47.8 & 47.8 \\ \text { No. at risk } & 18 & 7 & 2 & 2 & 2 & 2 \\ \% & 100 & 38.9 & 11.1 & 11.1 & 11.1 & 11.1\end{array}$

\section{Figure 2}

Kaplan-Meier survival curves of progression-free survival

(A) Intention-to-treat patients $(n=49)$. (B) Per-protocol patients (neoadjuvant treatment completion and tumor resection, $n=31$ ) and off-protocol patients (neoadjuvant failure or probe laparotomy, $n=18$ ). PFS, progression-free survival; MST, median survival time 
Figure 3

(A)

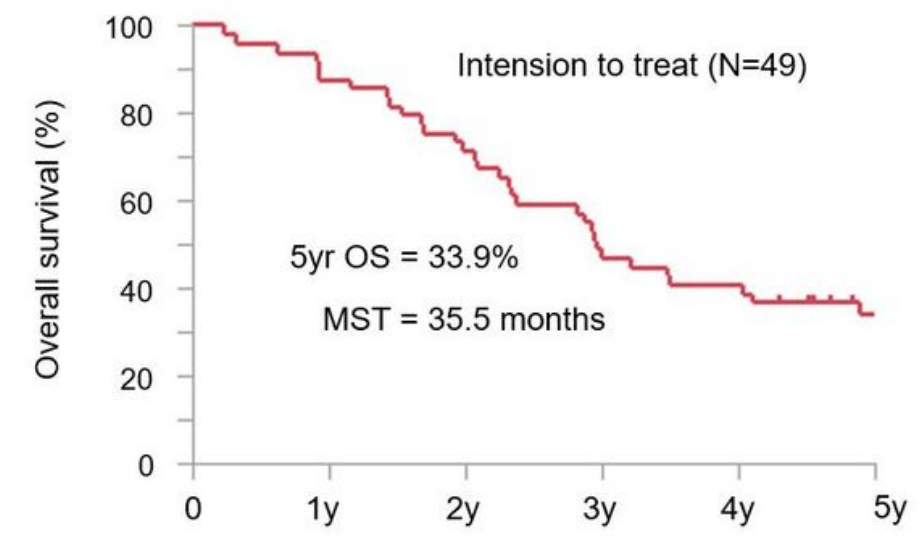

(B)

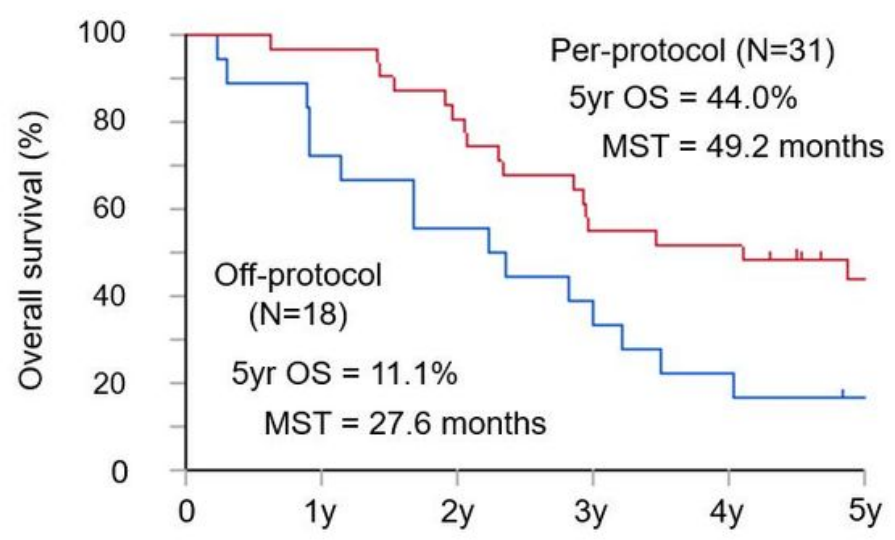

\section{2}

33.9
No. at risk 31

$\% \quad 100$

No. at risk 18

$\% \quad 100$
$30 \quad 25$

$\begin{array}{ll}96.8 & 80.7\end{array}$

17

16

54.8

$51.6 \quad 44.0$

$13 \quad 10$

$72.2 \quad 55.6$

7

38.9

42

$22.2 \quad 11.1$

\section{Figure 3}

Kaplan-Meier survival curves for overall survival

(A) Intention to treat patients $(n=49)$. (B) Per-protocol patients (neoadjuvant treatment completion and tumor resection, $\mathrm{n}=31$ ) and off-protocol patients (neoadjuvant failure or probe laparotomy, $\mathrm{n=18}$ ). OS, overall survival; MST, median survival time

\section{Supplementary Files}

This is a list of supplementary files associated with this preprint. Click to download.

- HOPSR01SupplementalTablee1e4.docx

- SupplementalFiguree1e3.pptx

- SupplementaryFiguree1e3legends.docx 